\title{
Gate-on-Drain Overlapped L-Shaped Channel Tunnel FET as Label-Free Biosensor
}

\author{
Suman Das ${ }^{1} \cdot$ Bikash Sharma $^{1}$ (D) \\ Received: 22 April 2021 / Accepted: 19 July 2021 / Published online: 24 July 2021 \\ (C) Springer Nature B.V. 2021
}

\begin{abstract}
In this work gate-on-drain L-shaped channel Tunnel FET is proposed to detect various biomolecules through label-free biosensing detection technique. Biomolecules can be detected in the proposed structure through modulation of ambipolar current between channel and drain. Modulation of ambipolar current is performed by extending gate over drain in order to create a gate to drain overlap (cavity) by etching the specific portion of the gate. Trapped biomolecules within cavity gets immobilized. Immobilized biomolecules change the drain to channel tunneling width, thus changing the ambiploar leakage current. Drain doping and cavity length was fine-tuned to achieve better sensitivity in terms of ambipolar current and ambipolar knee voltage shift with and without presence of biomolecules. A maximum sensitivity of $3.8 \times 10^{7}$ is achieved for drain doping of $5 \times 10^{19}$ donors $/ \mathrm{cm}^{3}$ and cavity length of $60 \mathrm{~nm}$. A high value of sensitivity is achieved for each biomolecules when drain doping ranged from $10^{19}$ donors $/ \mathrm{cm}^{3}$ to $5 \times 10^{19}$ donors $/ \mathrm{cm}^{3}$ and cavity length ranged between $40 \mathrm{~nm}$ to $50 \mathrm{~nm}$. Effect of differently charged biomolecules on sensitivity has also be structured.
\end{abstract}

Keywords Tunnel FET $\cdot$ Biosensor $\cdot$ Label free detection $\cdot$ Gate on drain overlap $\cdot$ Dielectric modulation $\cdot$ Ambipolar leakage

\section{Introduction}

Detection of biomolecules using biosensor has become a fastgrowing field of study in present COVID 19 pandemic situation. It creates enormous research interest among researchers to discover different approach to detect the biomolecules. Lots of attempt has been made to make biosensor [1,2]. Field effect transistor (FET) based biosensors (bio-FET) [3-11] are mostly drawing attention for its label free detection of biomolecules. The existence of biomolecules in free air change the properties of bio-FET such as current, threshold voltage, subthreshold swing, transconductance, etc. Apart from these advantages, bio-FET is compatible with CMOS technology which support system on chip configurations, smaller dimensions and used to detect biomolecules from the air, making change in channel conductance.

Bikash Sharma

ju.bikash@gmail.com

1 Department of Electronics and Communication Engineering, Sikkim Manipal Institute of Technology, Sikkim Manipal University, Sikkim 737136, India
Tunnel FET (TFET) is one of the emerging devices which overwriting the conventional MOSFET as a biosensor due to its band to band tunneling mechanism. It is overcoming the issue of sub- $60 \mathrm{mV} /$ decade subthreshold swing for MOSFET at room temperature and also TFET emerging as a promising candidate for various sensing application [11-16]. Dielectric modulated TFET with various structures [6-9, 11-16] have been reported since last decades which is able to produce higher sensitivity. The higher sensitivity in biosensors means

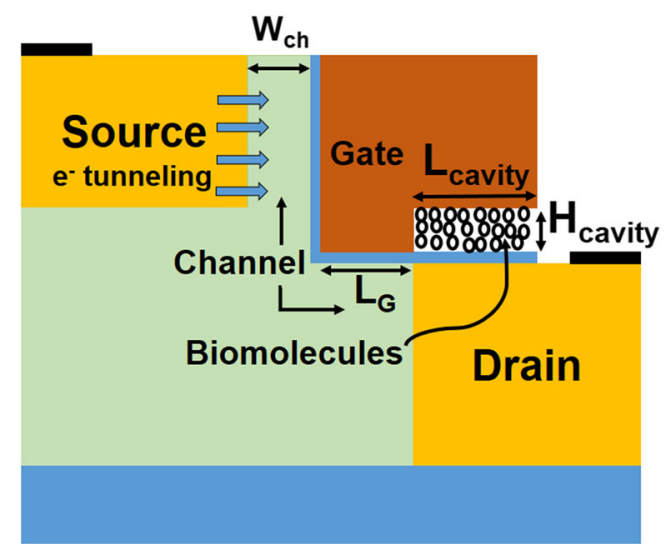

Fig. 1 Schematic of gate-on-drain overlapped L-shaped channel TFET (GDOL-TFET) 
Fig. 2 (a) ON state drain current of GDOL-TFET at $\mathrm{V}_{\mathrm{DS}}=1 \mathrm{~V}$ (b) Energy band profile of source channel junction at $\mathrm{V}_{\mathrm{GS}}=1 \mathrm{~V}$ and $\mathrm{V}_{\mathrm{DS}}=1 \mathrm{~V}$. (c) OFF state drain current of GDOL-TFET at $\mathrm{V}_{\mathrm{DS}}=1 \mathrm{~V}$ (d) Energy band profile of channel drain junction at $\mathrm{V}_{\mathrm{GS}}=-1 \mathrm{~V}$ and $\mathrm{V}_{\mathrm{DS}}=1 \mathrm{~V}$
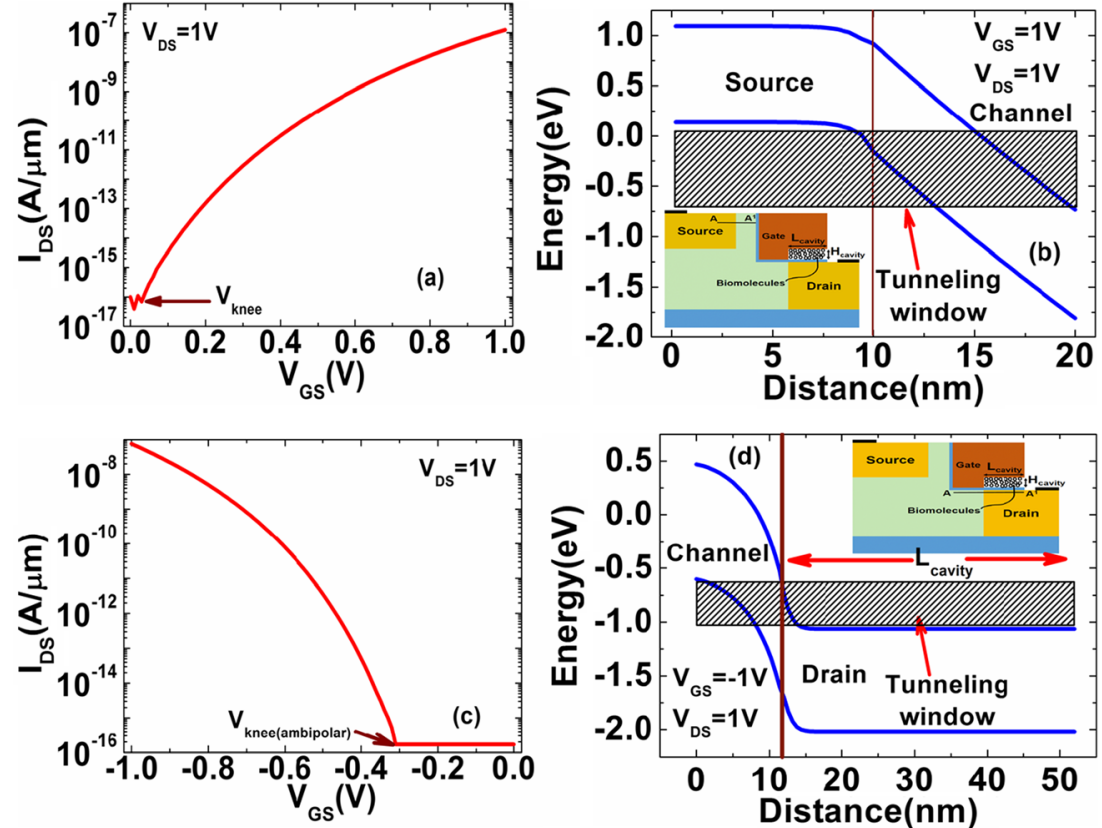

detectability of various biomolecules from air with a small change in electrical parameters. Lots of attempt has been made to increase the sensitivity of TFET based biosensor using either by material engineering or modulating the device structure. Most of the results reported by various authors based on drain current modulation for different biomolecules at positive gate voltage. In this literature an attempt has been made to design a silicon-based L-shaped channel TFET, following the experimental structure reported by Kim et al. [17], at state of art technology node [18]. Sensing of biomolecules have been achieved by modulating the ambipolar current between channel to drain at negative gate voltage by extending gate over drain thus making a cavity to trap the biomolecules. Various studies have been carried out to reduce the ambipolar leakage by overlapping gate on drain and increasing the dielectric constant of gate on drain overlap region [19]. Overlap between gate and drain, widens the tunneling barrier width at the channel-drain interface at negative gate voltages, which modulate the ambipolar current. In this manuscript for the first time a gate-on-drain overlapped L-shaped channel TFET (GDOL-TFET) has been reported to sense different biomolecules having dielectric constant between $\mathrm{K}=1$ to 10 which
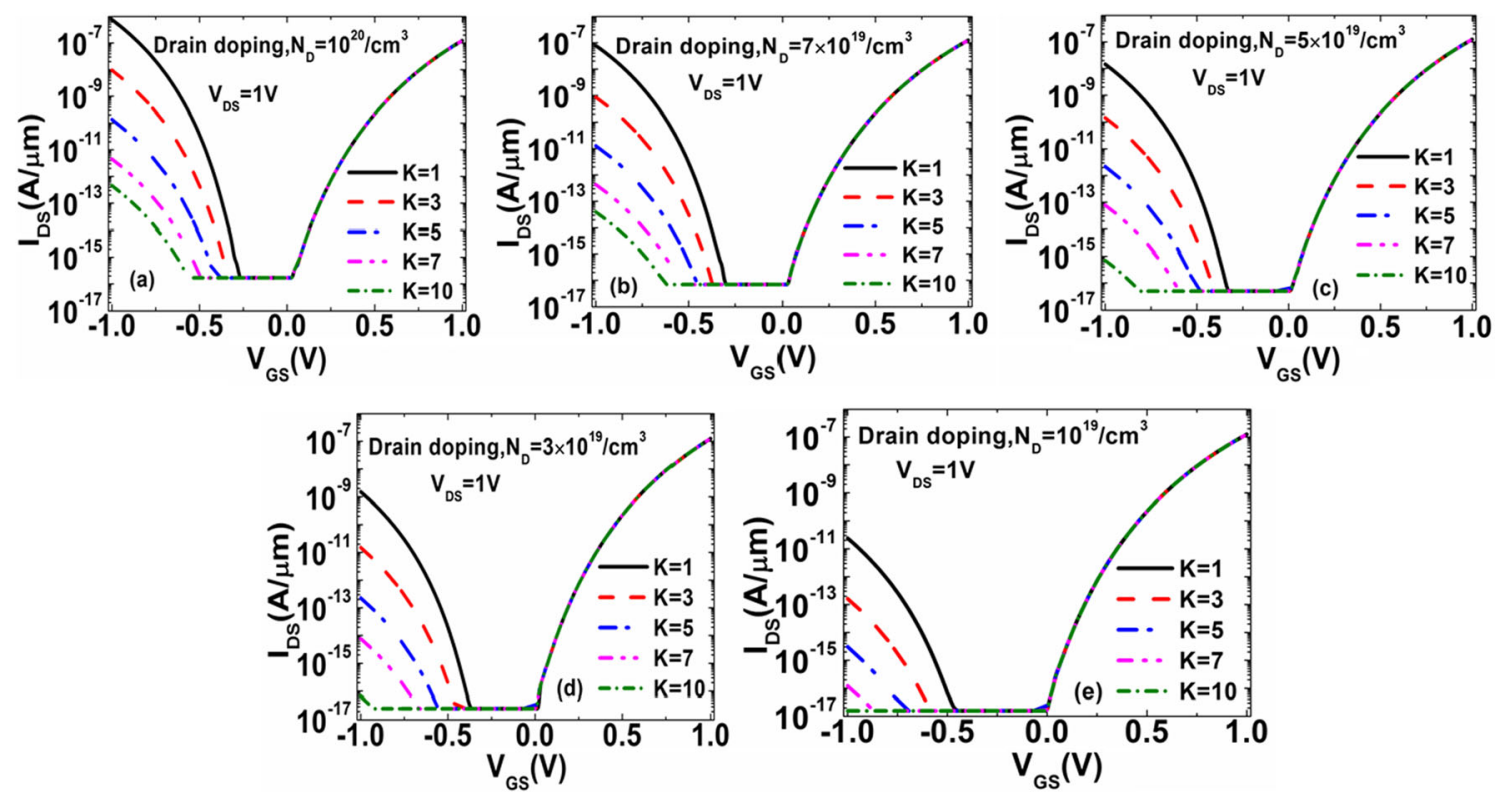

Fig. 3 Variation of ambipolar leakage current w.r.t. drain doping (a) $\mathrm{N}_{\mathrm{D}}=10^{20}$ donors $/ \mathrm{cm}^{3}$ (b) $\mathrm{N}_{\mathrm{D}}=7 \times 10^{19}$ donors $/ \mathrm{cm}^{3}(\mathbf{c}) \mathrm{N}_{\mathrm{D}}=5 \times 10^{19}$ donors $/ \mathrm{cm}^{3}$ (d) $\mathrm{N}_{\mathrm{D}}=3 \times 10^{19}$ donors $/ \mathrm{cm}^{3}$ (e) $\mathrm{N}_{\mathrm{D}}=10^{19}$ donors $/ \mathrm{cm}^{3}$ for different biomolecules 

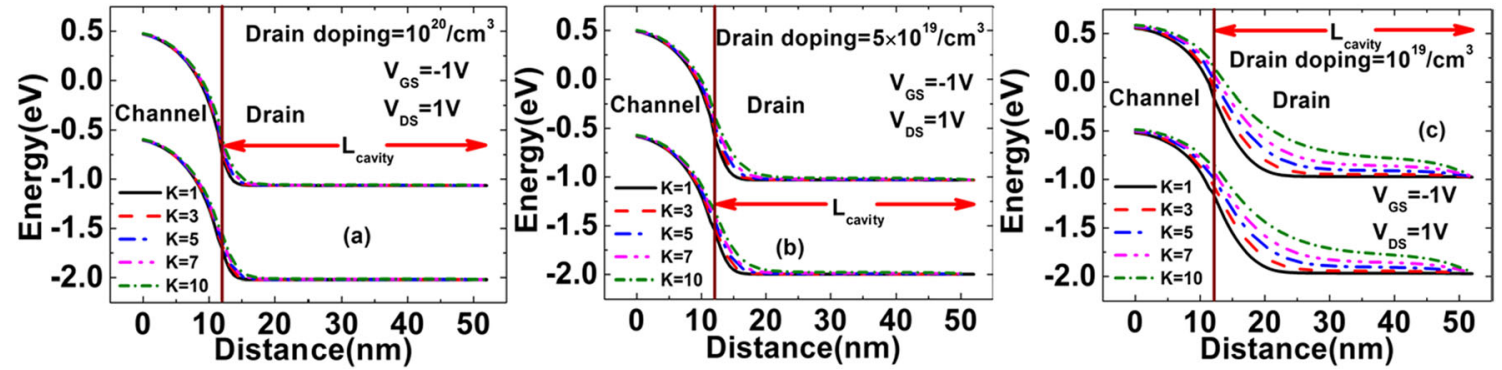

Fig. 4 Energy band diagrams of drain channel interface at different drain doping (a) $\mathrm{N}_{\mathrm{D}}=10^{20}$ donors $/ \mathrm{cm}^{3}$ (b) $\mathrm{N}_{\mathrm{D}}=5 \times 10^{19}$ donors $/ \mathrm{cm}^{3}(\mathbf{c}) \mathrm{N}_{\mathrm{D}}=10^{19}$ donors $/ \mathrm{cm}^{3}$ for different biomolecules

works on the phenomenon of reduction in ambipolar current due to gate to drain overlap.

\section{Device Structure, Operating Principle and Simulation Setup}

The schematic of gate-on-drain overlapped L-shaped TFET (GDOL-TFET) is shown in Fig. 1. Here an L shaped channel device structure is taken into consideration due to its vertical tunneling mechanism from source to channel which in turns increasing the band to band tunneling current rather than lateral tunneling device structures. The gate length is considered as $13 \mathrm{~nm}$ based on latest technology roadmap. The width of channel $\left(\mathrm{W}_{\mathrm{ch}}\right)$ and thickness of oxide $\left(\mathrm{SiO}_{2}\right)$ are considered as $10 \mathrm{~nm}$ and $0.6 \mathrm{~nm}$ respectively. The work function of gate metal is chosen as $4.1 \mathrm{eV}$. One cavity is created to immobilize the biomolecules between gate and drain, which have length $\mathrm{L}_{\text {cavity }}=40 \mathrm{~nm}$ and height $\mathrm{H}_{\text {cavity }}=10 \mathrm{~nm}$ respectively. This cavity is used to decrease the ambipolar leakage current as well as is used to detect the biomolecules. A $0.6 \mathrm{~nm}$ thick $\mathrm{SiO}_{2}$ is introduced throughout cavity to minimize the leakage current as well as uniform doping concentration of $\mathrm{P}+$ source, $\mathrm{N}$ channel and $\mathrm{N}+$ drain are considered with doping concentration of $10^{20}$ acceptors $/ \mathrm{cm}^{3}, 10^{17}$ donors $/ \mathrm{cm}^{3}$ and $10^{20}$ acceptors $/ \mathrm{cm}^{3}$ respectively.

The operation of the device is shown in Fig. 1. The operation depends on vertical electron tunneling from the valance band of source to the conduction band of channel at gate to source voltage $\left(\mathrm{V}_{\mathrm{GS}}\right)$ greater than the knee voltage $\left(\mathrm{V}_{\text {knee }}\right)$ of the device. $\mathrm{V}_{\mathrm{knee}}$ is the minimum gate to source voltage required for the device to enter into sub-threshold region. At $\mathrm{V}_{\mathrm{GS}}>\mathrm{V}_{\mathrm{knee}}$ the energy band of channel region start to bend and the valance band of source start to overlap with the conduction band of channel thus creating a tunneling window, which will be responsible for electron flow from source to channel. This band to band tunneling depends on the tunneling width and tunneling area of the tunneling junction between source to channel. Tunneling junction area depends on the gate to source overlap and width of the vertical channel $\left(\mathrm{W}_{\mathrm{ch}}\right)$ region. After tunneling, electrons will accumulate in the channel-oxide interface which will be swept away by the drain voltage to the drain region thus rising a drain current between drain to source. The drain current and its corresponding energy band diagram is shown in Fig. 2(a) and (b) which defines the operation.

But the main concern with this structure is the ambipolar leakage which generate at positive $\mathrm{V}_{\mathrm{DS}}$ and negative $\mathrm{V}_{\mathrm{GS}}$. At negative $V_{G S}$ the energy band of channel under gate region will move upward and at positive $\mathrm{V}_{\mathrm{DS}}$ the energy band of drain region will move downward. At a particular gate bias, $\mathrm{V}_{\mathrm{GS}}=\mathrm{V}_{\text {knee(ambipolar) }}$ the valance band of channel start to overlap conduction band of drain as a result electron start to tunnel through the tunneling window and a tunneling current can be obtained which is undesirable for digital circuit application where leakage current should be minimum. The drain current and its corresponding energy band diagram is shown in Fig. 2(c) and (d) which state the operation.

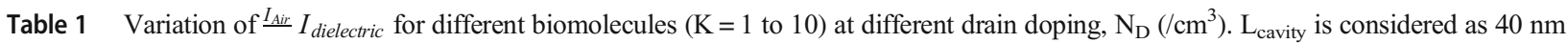

\begin{tabular}{|c|c|c|c|c|c|}
\hline \multirow[t]{2}{*}{ Drain doping, $\mathrm{N}_{\mathrm{D}}\left(/ \mathrm{cm}^{3}\right)$} & \multicolumn{5}{|c|}{$\underline{I_{\text {Air }}} I_{\text {dielectric }}$} \\
\hline & $\mathrm{K}=1$ & $K=3$ & $K=5$ & $K=7$ & $K=10$ \\
\hline $10^{19}$ & 1 & 150 & 7857 & $2 \times 10^{5}$ & $1.55 \times 10^{6}$ \\
\hline $3 \times 10^{19}$ & 1 & 120 & 7100 & $1.95 \times 10^{5}$ & $2.23 \times 10^{7}$ \\
\hline $5 \times 10^{19}$ & 1 & 100 & 6700 & $1.86 \times 10^{5}$ & $2.07 \times 10^{7}$ \\
\hline $7 \times 10^{19}$ & 1 & 90 & 6100 & $1.79 \times 10^{5}$ & $1.95 \times 10^{7}$ \\
\hline $10^{20}$ & 1 & 80 & 5600 & $1.7 \times 10^{5}$ & $1.7 \times 10^{6}$ \\
\hline
\end{tabular}


Table 2 Variation of $\mathrm{V}_{\text {knee }}$ for different biomolecules $(\mathrm{K}=1$ to 10$)$ at different drain doping, $\mathrm{N}_{\mathrm{D}}\left(/ \mathrm{cm}^{3}\right)$. $\mathrm{L}_{\text {cavity }}$ is considered as $40 \mathrm{~nm}$

\begin{tabular}{|c|c|c|c|c|c|}
\hline \multirow[t]{2}{*}{ Drain doping, $\mathrm{N}_{\mathrm{D}}\left(/ \mathrm{cm}^{3}\right)$} & \multicolumn{5}{|l|}{$\mathrm{V}_{\text {knee }}$} \\
\hline & $\mathrm{K}=1$ & $\mathrm{~K}=3$ & $\mathrm{~K}=5$ & $\mathrm{~K}=7$ & $\mathrm{~K}=10$ \\
\hline $10^{19}$ & 0 & 0.13 & 0.26 & 0.42 & 0.54 \\
\hline $3 \times 10^{19}$ & 0 & 0.09 & 0.19 & 0.31 & 0.56 \\
\hline $5 \times 10^{19}$ & 0 & 0.07 & 0.16 & 0.26 & 0.48 \\
\hline $7 \times 10^{19}$ & 0 & 0.04 & 0.14 & 0.24 & 0.32 \\
\hline $10^{20}$ & 0 & 0.05 & 0.13 & 0.22 & 0.29 \\
\hline
\end{tabular}

All simulation results are obtained by Silvaco Atlas, version 3.20.2.R [20]. Non-local band to band tunneling are taken into account to obtain the band to band tunneling between source to channel. To incorporate mobility effect concentration dependent mobility model and CVT Lombardi model are triggered. Due to high doping in source and drain region band gap narrowing model is enabled. To get the recombination effect Shockley-Read-Hall recombination is used with Fermi-Dirac distribution statistics.

\section{Results and Discussions}

Minimization of ambipolar leakage current was carried out by different authors in last decade [21-23]. Out of which one method to reduce the ambipolar leakage is overlapping the gate on drain [19]. Overlap between gate and drain increases the capacitive coupling between gate and drain thus reducing the ambipolar current. Gate on drain overlapped tunnel FET as bio sensing application was first reported by abdi. et al. [24]. But here for the first time gate-on-drain overlapped method is applied to L-shaped tunnel FET for bio sensing application.

Different biomolecules have different dielectric constant. Based on this fact cavity is filled with different biomolecules with different dielectric constant and ambipolar current is measured each time. Sensitivity of this device is obtained by i) $I_{D \text {,air }} / I_{D \text {,biomolecules }}$ where $I_{D \text {,air }}$ is the ambipolar current when cavity is filled with air and $\mathrm{I}_{\mathrm{D} \text {,biomolecules }}$ is the ambipolar drain current when cavity is filled with biomolecules ii) $\mathrm{V}_{\text {knee,ambiploar }}$ shift between $\mathrm{V}_{\text {knee,ambiploar }}$ of air in cavity and $\mathrm{V}_{\text {knee,ambiploar }}$ of biomolecules in cavity. Results section is divided with three portions viz. i) Optimization of drain doping w.r.t sensitivity ii) Optimization of cavity length w.r.t sensitivity. iii) Effect of positive and negative charged biomolecules in cavity.

\subsection{Optimization of Drain Doping}

Doping concentration of drain region influence the sensitivity of GDOL-TFET as biosensor. Figure 3 shows the variation of ambipolar leakage current for different drain doping under the influence of different biomolecules in cavity. Dielectric constant of biomolecules are taken as $\mathrm{K}=1,3,5,7,10$. From Fig. 3(a) one can notice that as the dielectric constant is increasing,

Fig. 5 Variation of sensitivity at different drain doping for different biomolecules (a) sensitivity w.r.t. current (b) sensitivity w.r.t. knee voltage shift. $\mathrm{L}_{\text {cavity }}$ is considered as $40 \mathrm{~nm}$
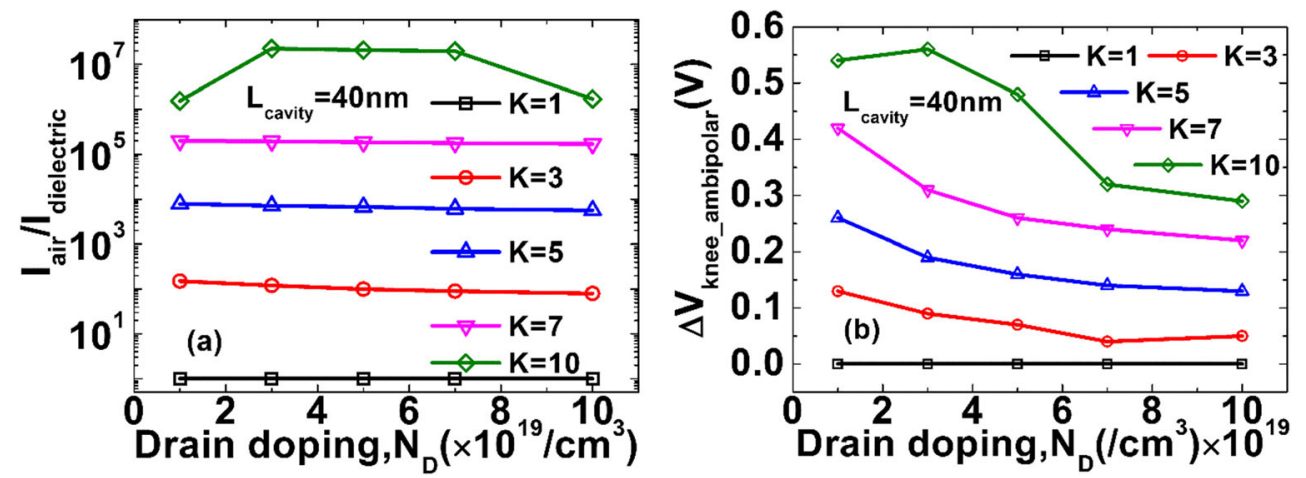

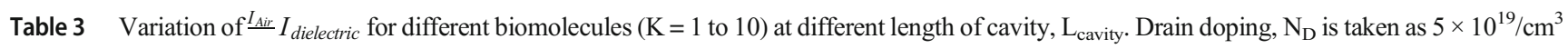

\begin{tabular}{|c|c|c|c|c|c|}
\hline \multirow{2}{*}{$\begin{array}{l}\mathrm{L}_{\text {cavity }} \\
(\mathrm{nm})\end{array}$} & \multicolumn{5}{|c|}{$\underline{I_{\text {Air }}} I_{\text {dielectric }}$} \\
\hline & $K=1$ & $K=3$ & $K=5$ & $\mathrm{~K}=7$ & $K=10$ \\
\hline 40 & 1 & 100 & 6700 & $1.86 \times 10^{5}$ & $2.07 \times 10^{7}$ \\
\hline 50 & 1 & 133 & 7800 & $2.02 \times 10^{5}$ & $3.05 \times 10^{7}$ \\
\hline 60 & 1 & 171 & 8600 & $2.2 \times 10^{5}$ & $3.87 \times 10^{7}$ \\
\hline
\end{tabular}


Table 4 Variation of $\mathrm{V}_{\text {knee }}$ for different biomolecules $(\mathrm{K}=1$ to 10$)$ at different length of cavity, $\mathrm{L}_{\text {cavity. }}$. Drain doping, $\mathrm{N}_{\mathrm{D}}$ is taken as $5 \times 10^{19}$ / $\mathrm{cm}^{3}$

\begin{tabular}{|c|c|c|c|c|c|}
\hline \multirow{2}{*}{$\begin{array}{l}\mathrm{L}_{\text {cavity }} \\
\text { (nm) }\end{array}$} & \multicolumn{5}{|l|}{$\mathrm{V}_{\text {knee }}$} \\
\hline & $K=1$ & $\mathrm{~K}=3$ & $K=5$ & $K=7$ & $\mathrm{~K}=10$ \\
\hline 40 & 0 & 0.07 & 0.16 & 0.26 & 0.48 \\
\hline 50 & 0 & 0.09 & 0.19 & 0.31 & 0.57 \\
\hline 60 & 0 & 0.1 & 0.21 & 0.33 & 0.63 \\
\hline
\end{tabular}

the capacitive coupling between drain and gate throughout the cavity length is increasing which resulting in less band bending in drain region and wider tunnel width in drain-channel interface. Wider tunnel width means less tunnel current and reduction in ambipolar leakage. The band bending for different $\mathrm{K}$ values at drain doping, $\mathrm{N}_{\mathrm{D}}=10^{20}$ donors $/ \mathrm{cm}^{3}$ is shown in Fig. 4(a). From Fig.3(b)-(e) it is clear that for a particular K value as the doping is decreasing the ambipolar leakage is decreasing and the $V_{\text {knee(ambipolar) }}$ is shifting towards more negative value. This phenomenon can be explained by energy band diagram of channel drain interface for $\mathrm{V}_{\mathrm{GS}}=-1 \mathrm{~V}$ and $\mathrm{V}_{\mathrm{DS}}=1 \mathrm{~V}$ as shown in Fig. 4(a)-(c). For a particular $\mathrm{K}$ value as the drain doping is decreasing the drain energy band throughout the cavity is bending less and tunneling width is increasing which producing less ambipolar leakage current.

Sensitivity is calculated through $\frac{I_{\text {air }}}{I_{\text {dielectric }}}$ ratio and $\mathrm{V}_{\text {knee(ambipolar) }}$ shift, $\Delta \mathrm{V}_{\text {knee(ambipolar) }}$ as shown in Fig. 5,
Tables 1 and 2. From Fig. 5(a) and Table 1 it is clear that for highly doped drain region the sensitivity is less for a particular value of $\mathrm{K}$. When doping is decreased the sensitivity is increasing. Highest sensitivity is achieved for $\mathrm{K}=10$ and drain doping $=3 \times 10^{19}$ donors $/ \mathrm{cm}^{3}$. From Fig. $5(\mathrm{~b})$ and Table 2 the same trend is noticed throughout the range of K. For low doped drain the $V_{\text {knee(ambipolar) }}$ shift is high whereas for high doping $\mathrm{V}_{\text {knee(ambipolar) }}$ shift is low. Maximum $\mathrm{V}_{\text {knee(ambipolar) }}$ shift is achieved for $\mathrm{K}=10$ and drain doping $=3 \times 10^{19}$ donors $/ \mathrm{cm}^{3}$. But if we concentrate on overall trend of both Fig. 5(a) and (b) one can understand that for the range of doping from $10^{19}$ donors $/ \mathrm{cm}^{3}$ to $5 \times 10^{19}$ donors $/ \mathrm{cm}^{3}$ the $\frac{I_{\text {air }}}{I_{\text {dielectric }}}$ ratio and $V_{\text {knee(ambipolar) }}$ shift is well enough to detect different biomolecules ranging from $\mathrm{K}$ value 3 to 10 . Drain doping cannot be decreased below $10^{19}$ donors $/ \mathrm{cm}^{3}$ as depicted from Fig. 3(e). It is justifying that at this doping the ambipolar current of $\mathrm{K}=7$ and $\mathrm{K}=10$ will be same and the device will not be able to detect the difference between these two biomolecules.

\subsection{Optimization of $\mathrm{L}_{\text {cavity }}$}

Effect of length of cavity, $\mathrm{L}_{\text {cavity }}$ on ambipolar current for different biomolecules present within cavity is shown in Fig. 6. From Fig. 6 one can observe that, for a particular value of $\mathrm{K}$ when cavity length increases, the overlap between cavity and drain also increases. The increased cavity area will trap more and more biomolecules within the cavity. These immobilized biomolecules will affect the band bending of drain region and
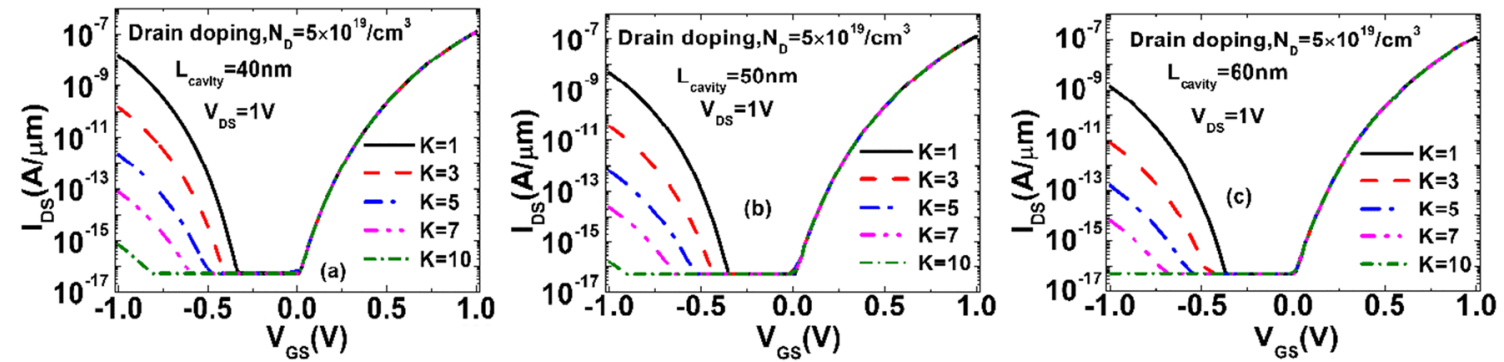

Fig. 6 Variation of ambipolar leakage current w.r.t. $\mathrm{L}_{\text {cavity }}(\mathbf{a}) \mathrm{L}_{\text {cavity }}=40 \mathrm{~nm}(\mathbf{b}) \mathrm{L}_{\text {cavity }}=50 \mathrm{~nm}(\mathbf{c}) \mathrm{L}_{\text {cavity }}=60 \mathrm{~nm}$ for different biomolecules

Fig. 7 Variation of sensitivity at different value of $\mathrm{L}_{\text {cavity }}$ for different biomolecules (a) sensitivity w.r.t. current (b) sensitivity w.r.t. knee voltage shift
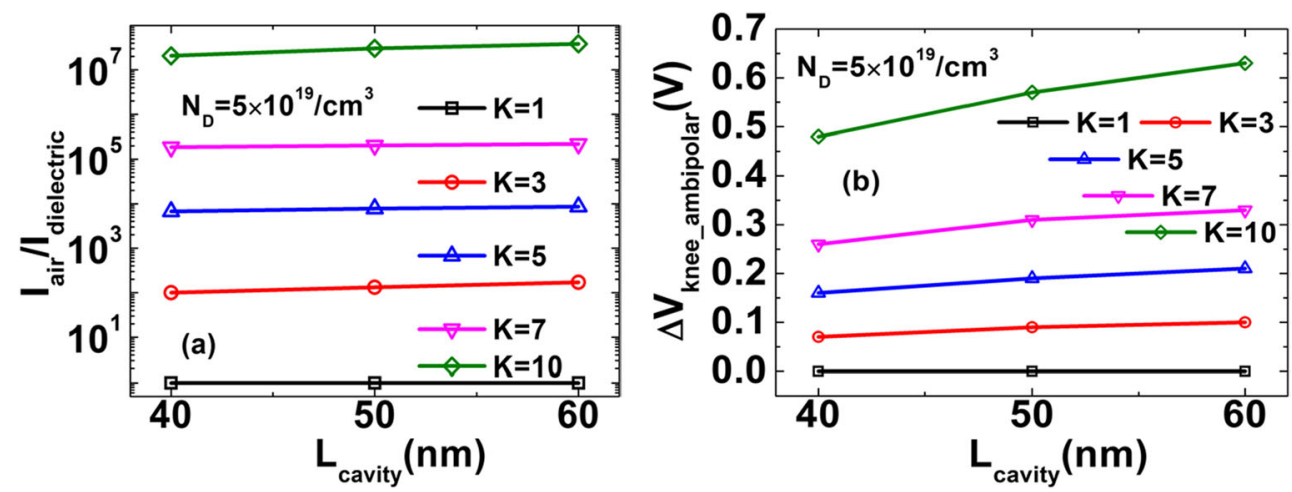
Fig. 8 Variation of sensitivity w.r.t various positive and negative charge density for different biomolecules (a) positively charged biomolecules (b) negatively charged biomolecules

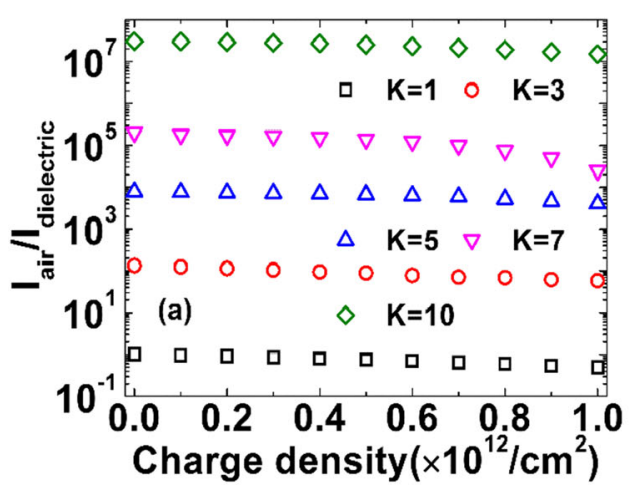

tunneling width between channel and drain will increase thus generating less ambipolar drain current. Figure 7, Tables 3 and 4 shows the sensitivity w.r.t. $\mathrm{L}_{\text {cavity }}$ for different biomolecules at drain doping, $\mathrm{N}_{\mathrm{D}}=5 \times 10^{19} / \mathrm{cm}^{3}$. From Fig. 7, Tables 3 and 4 it is clear that for larger value of $\mathrm{L}_{\text {cavity }}, \frac{I_{\text {air }}}{I_{\text {dielectric }}}$ ratio and $\mathrm{V}_{\mathrm{knee}(\mathrm{ambipolar})}$ shift is increasing. But designer should keep in mind that increasing $\mathrm{L}_{\text {cavity }}$ means increments in total gate length also, which is not feasible all the time at the edge of device miniaturization. Increase in $\mathrm{L}_{\text {cavity }}$ by $10 \mathrm{~nm}$ is not significantly improving the $\frac{I_{a i r}}{I_{\text {dielectric }}}$ ratio and $\mathrm{V}_{\text {knee(ambipolar) }}$ shift. So it will be justified if we keep the $\mathrm{L}_{\text {cavity }}$ in between $40 \mathrm{~nm}$ to $50 \mathrm{~nm}$.

\subsection{Effect of Positive and Negative Charged Biomolecules in Cavity}

The effect of positive (p-type) and negative charge (n-type) immobilized in cavity will either improve or degrade the sensitivity of the biosensor. In the proposed structure sensitivity depends on the drain depletion. Negatively charged biomolecules depletes the $\mathrm{N}^{+}$drain region resulting in better sensitivity, whereas positively charged biomolecules shows the opposite effect. Figure 8 depicts the variation of sensitivity with respect to various positive and negative charge density for different biomolecules.

\section{Conclusion}

In this manuscript we have proposed a gate-on-drain L-shaped channel Tunnel FET for label free biosensor based on modulation of ambipolar current depending on the cavity between drain and gate. Sensitivity was varied depending on drain doping, length of cavity and charge density of biomolecules. Drain doping is optimized at a range of $10^{19}$ donors $/ \mathrm{cm}^{3}$ to $5 \times 10^{19}$ donors $/ \mathrm{cm}^{3}$. Highest sensitivity is achieved for $\mathrm{K}=$ 10 and drain doping $=3 \times 10^{19}$ donors $/ \mathrm{cm}^{3}$. Length of cavity is proposed to be optimized at $40 \mathrm{~nm}$ to $50 \mathrm{~nm}$. Also depending on the charged density for different biomolecules sensitivity is either increased or decreased, which is well established in our manuscript.

Acknowledgements

Authors Would Like to Thank AICTE, Govt. of India and SMIT, SMU for the Support of the Work.Authors' Contributions Both the authors have contributed equally.

Funding This work was supported by All India Council for Technical Education (AICTE) Govt. of Indiaunder Research Promotion Scheme for North-East Region (RPS-NER) vide ref.: File No. 8-139/RIFD/RPSNER/Policy-1/2018-19.

Data Availability Yes

\section{Declarations}

Ethics Approval and Consent to Participate This is an original work by the authors and the results presented are true as achieved. This workhas not been submitted elsewhere in any form or language.

Informed Consent Not Applicable.

Consent for Publication Yes

Conflict of Interest Not Applicable.

Competing Interests The work is a part of the sponsored project mentioned in the funding. It has been carried out atSMIT, where both the authors are faculty in the ECE Dept.

Research Involving Human Participants and/or Animals Not Applicable.

\section{References}

1. Mehrotra P (2016) Biosensors and their applications-a review. J Oral Biol Craniofacial Res 6(2):153-159

2. Kougianos E (2006) Biosensors : a tutorial review. IEEE Potentials 25(2):35-40

3. Narang R, Sasidhar Reddy KV, Saxena M, Gupta RS, Gupta M (2012) A dielectric-modulated tunnel-fet-based biosensor for label- 
free detection: analytical modeling study and sensitivity analysis. IEEE Trans Electron Devices 59(10):2809-2817

4. Sarkar D, Banerjee K (2012) Fundamental limitations of conventional-FET biosensors: quantum-mechanical-tunneling to the rescue. Device Res Conf - Conf Dig DRC:83-84

5. Kanungo S, Chattopadhyay S, Gupta PS, Sinha K (2016) Study and analysis of the effects of SiGe source and pocket doped channel on sensing performance of dielectrically modulated tunnel FET based biosensor. IEEE Trans Electron Devices 63(6):2589-2596

6. Kanungo S, Chattopadhyay S, Gupta PS, Rahaman H (2015) Comparative performance analysis of the dielectrically modulated full-gate and short-gate tunnel FET-based biosensors. IEEE Trans Electron Devices 62(3):994-1001

7. Narang R, Saxena M, Gupta M (2015) Comparative analysis of dielectric- modulated FET and TFET based biosensor. IEEE Trans Nanotechnol 14(3):427-435

8. Ghosh B, Akram MW (2013) Junction less tunnel field-effect transistor. IEEE Electron Device Lett 34(5):584-586

9. Chandan VB, Nigam K, Sharma D (2018) Junctionless based dielectric modulated electrically doped tunnel FET based biosensor for label-free detection. IET Circuits, Devices Syst 13(4):452-456

10. Sarkar D, Banerjee K (2012) Proposal for tunnel-fieldeffecttransistor as ultra-sensitive and label-free biosensors. Appl Phys Lett 100(14): 143108

11. Im H, Huang XJ, Gu and Choi Y K 2007 A dielectric-modulated field-effect transistor for biosensing. Nature Nanotech 2:430-434

12. Verma M, Tirkey S, Yadav S, Sharma D, Yadav DS (2017) Performance assessment of a novel vertical dielectrically modulated TFET-based biosensor. IEEE Tran on Elec Dev 64(9):38413848

13. Kumar S, Singh Y, Singh B, Tiwari PK (2020) Simulation study of dielectric modulated dual channel trench gate TFET-based biosensor. IEEE Sensors J 20(21):12565-12573

14. Venkatesh P, Nigam K, Pandey S, Sharma D, Kondekar PN (2017) A dielectrically modulated electrically doped tunnel FET for application of label free biosensor. Superlattice Microst 109:470-479
15. Dwivedi P, Singh R (2020) Investigation the impact of the gate work-function and biases on the sensing metrics of TFET based biosensors. Eng Res Express 2(2):025043

16. Singh D, Pandey S, Nigam K, Sharma D, Yadav DS, Kondekar P (2017) A charge-plasma-based dielectric-modulated Junctionless TFET for biosensor label-free detection. IEEE Trans on Elec Dev 64(1):271-278

17. Kim SW, Kim JH, Liu TK, Choi WY, Park B (2016) Demonstration of L-shaped tunnel field-effect transistors. IEEE Tran Elec Dev 63(4):1774-1778

18. The International Technology Roadmap for Semiconductors (ITRS) (2015) [Online]. Available: http://www.itrs2.net

19. Abdi DB, Jagadesh Kumar M (2014) Controlling Ambipolar current in tunneling FETs using overlapping gate-on-drain. IEEE J Electron Devices Soc 2(6):187-190

20. SILVACO Inc (2016) ATLAS user's manual," A 2-D Device Simulator Software Package, Santa Clara, CA, USA, [Online]. Available: http://www.silvaco.com

21. Ashita LSA, Alharbi AG, Rafat M (2018) Ambipolar leakage suppression in electron-hole bilayer TFET: investigation and analysis. J Comput Electron 17:977-985

22. Hu VPH, Wang CT (2018) Optimization of III-V heterojunction tunnel FET with non-uniform channel thickness for performance enhancement and ambipolar leakage suppression. Jpn J Appl Phys 57(4S):04FD18

23. Imenabadi RM, Saremi M, Vandenberghe WG (2017) A novel PNPN-like Z-shaped tunnel field- effect transistor with improved Ambipolar behavior and RF performance. IEEE Tran on Elec Dev 64(11):4752-4758

24. Abdi DB, Kumar MJ (2015) Dielectric modulated overlapping gate-on-drain tunnel-FET as a label-free biosensor. Superlattice Microst 86:198-202

Publisher's Note Springer Nature remains neutral with regard to jurisdictional claims in published maps and institutional affiliations. 